Feature

\title{
Inclusive or Exclusive? Body Positive Communication in Imagery and Clothing in Athens, Greece
}

Ashley Camarneiro*

\begin{abstract}
It is expected that $40 \%$ of adults in Greece will be obese by 2030 , and more and more individuals in the country are inactive. There are many reasons for obesity and inactivity; however, factors such as societal influences and appearance-focused communication are known to have an effect. Negative body communication-in other words, size-discrimination or shaming - may cause a person to consume unhealthy or large amounts of food and avoid exercise. Likewise, a system of social marking divides one group, the "ideal" group, from another group, the "lesser" group, thereby creating a perception of abnormality towards the "lesser" group and strengthening a social divide. Moreover, labelling theory states that individuals tend to behave based on the label assigned to them. In contrast, body positive communication seeks to challenge beauty standards and encourage a healthy mindset that in turn inspires healthy consumption and activity. This study analyzes communication towards females in Athens, Greece, through imagery, by examining front-of-store signage and mannequins, and clothing, by reviewing the size range available for purchase in stores. Major findings reveal that images do not show diversified sizing and the most common sizes are medium and small. This paper shows that negative communication could potentially exacerbate the overweight and obesity rate and that Athens, Greece has inadequate body positive communication practices.

Keywords: body size, body positive, communication, ideal body size, body shaming, clothing stores, clothing sizes, plus size
\end{abstract}




\section{Introduction}

Nearly half of Greece's population is overweight and obese with low engagement in physical activity; the obesity rate is estimated to rise (World Health Organization, 2013, p. 1, 3). Size-discriminatory communication-in other words, body shaming-has negative effects on women including the development of eating disorders, increased eating or poor eating habits, and self-esteem issues (Aronson, Wilson, Fehr, \& Akert, 2013, pp. 216-217; Mann, Tomiyama, \& Ward, 2015, pp. 706-710; Tomiyama \& Mann, 2013, pp. 45). Other factors, such as poor stress management, also contribute to unhealthy eating habits; in fact, one in four Americans use food as a way to ease stress or manage their problems (American Psychological Association, 2006, Comfort eating, para. 1).

At the same time, humans are taught culturally appropriate behaviour through informational social influence, and social marking, a system that divides groups and implies one group is ideal and the other is lesser, shows that the impression of abnormality is increased through the use of unmarked and marked categories: unmarked being the "ideal," "normal" group and marked being the "lesser," "subset" group (Aronson et al., 2013, pp. 216-217; Brekhus, 1996, p. 497, 500). This means if imagery shows only unmarked categories and size selection in stores only contain sizes for unmarked categories, individuals are in the presence of size-discriminatory communication. In addition, labelling theory adds that a person may behave in a manner consistent to a label they have been assigned (Murray, Linden, \& Kendell, 2011, p. 176). Thus, the communication used to discuss body size and body size labels such as fat can also impact body size, i.e. if a person begins acting according to the stereotypical version of their label.

The media are currently changing the ideal body size narrative through a body positivity movement; this movement is important in communicating to women that size does not determine a person's self-worth (Dunn, as cited in Schreiber \& Hausenblah, 2016, "How do you", para. 1; Schreiber \& Hausenblas, 2016, "How do you", para. 2-3). It is unclear if this movement has had an impact in Athens, Greece or if size-inclusive communication is present in images displayed in shopping areas and clothing available in stores. This research seeks to demonstrate the importance of body positive communication and responds to the following question: To what degree is body positivity communicated to women in Athens, Greece, through imagery and clothing in stores?

\section{Limitations}

Given that the direct human interaction was not part of the research design, the researcher fulfilled the role as a shopper. Without being able to communicate with a 


\section{A. Camarneiro}

representative from the store, the researcher was required to appear as a shopper. This meant that, at times, the researcher needed to study sizes by moving naturally from rack to rack rather than using the systematic randomized approach as described in the methodology section. This also meant that the researcher was required to rely on the store tags, prior knowledge of the measurements, and visual observations to draw conclusions compared to just using a measuring tape.

An additional limitation was time. The researcher was in Athens, Greece, for a week and collected data over four days. As well, it was a conscious decision by the researcher to study women instead of studying all genders. This limit was set because accounting for the multitude of gender-differences regarding body and food would convolute this study. In addition, although the body positivity movement is genderinclusive, body-inclusive advertisements are generally directed towards women, and physical attractiveness is proven to be more important to women than to men (CwynarHorta, 2016, p. 37; Gottschall; Li \& Kenrick, as cited in Kościński, 2013, p. 914).

Last, while the researcher acknowledged similar challenges faced with those who are under the ideal weight, this study primarily focused on individuals over the ideal weight, specifically overweight or obese persons.

\section{Key Terms}

The following is a list of important terms or concepts relevant to this research study.

Body positive communication. Any form of communication that confronts the current perception of societal ideal beauty standards and encourages people to govern their own self-worth; it "encompasses any individual or movement actions which aim to denounce the societal influences and construction of body norms, and instead promotes self-love and acceptance of bodies of any shape, size, or appearance; including rolls, dimples, cellulite, acne, hairy bodies, bleeding bodies, fat bodies, thin bodies, and (dis)abled bodies" (Cwynar-Horta, 2016, p. 37). Such communication is size-inclusive and encourages choice and healthiness; it opposes an individual striving for a body size that is unattainable for the individual's body or capacity (p. 37). The main message is that women should have confidence in their unique form regardless if such form defies societal beauty norms.

Body positive imagery. Imagery that uses body positive communication; more specifically, imagery that includes "rolls, dimples, cellulite, acne, hairy bodies, bleeding 


\section{A. Camarneiro}

bodies, fat bodies, thin bodies, and (dis)abled bodies" (Cwynar-Horta, 2016, p. 37); includes size-inclusive imagery, as defined below.

Body positivity movement. Throughout this study, the body positive movement was sometimes referred to as "this movement." For this study, a movement is both "a group of people who share the same ideas or aims" and "a gradual change in what people in society do or think" (Movement, 2017, para 3, 5). The body positivity movement is a movement that "address[es] unrealistic ideals about beauty, promote[s] self-acceptance, and build[s] self esteem" (Cwynar-Horta, 2016, p. 37). This movement encourages individuals to accept their body including "changes in shape, size, and ability" that may occur "due to nature, age, and... [ones] own personal choices throughout... [their] lifetime" (Dunn, as cited in Schreiber \& Hausenblas, 2016, "How do you", para. 1). Further, this movement emphasizes that a person's appearance does not define self-worth and encourages the abolishment of self-hate (Dunn, as cited in Schreiber \& Hausenblah, 2016, "How do you", para. 1).

Ideal body size(s). In this study, "ideal body size" referred to the female body and is the socially constructed bodily appearance that is deemed to be most beautiful and held as the standard for women to achieve (Aronson et al., 2013, pp. 214-215). In Western cultures, specifically North American culture, thinness is what is valued in the female form (p. 215). However, "many, if not most, world societies consider plumpness in females attractive" (p. 215).

Informational social influence. "The mechanism by which women learn what kind of body is considered to be attractive at a given time in their culture" (Aronson et al., 2013 , p. 216). Women can learn this information through comparisons of family, friends, and the media (p. 216).

Labelling theory. A sociological theory that suggests deviance is a by-product of individuals being labelled as deviants by others, meaning individuals will behave consistent with a label assigned to them, even if it dissents from the norm; it is "related to the power and status of those persons who do the labelling and those who are being labelled"; deviants are "people who act in a manner contrary to expected" (Murray, Linden, \& Kendell, 2011, p. 176).

Normative social influence. Influence from others that encourages conformity in order to be liked and accepted; such influence usually results in compliance (Aronson et al., 2013, p. 202). 
Size-inclusive imagery. This is defined as imagery that includes a variety of body sizes. This definition was created by the researcher after viewing size-inclusive advertisements such as the recent advertisements by Dove, Aeire, and Old Navy that show images of diverse body sizes (Dubecky, n.d., para. 6-7, 9; Schreiber \& Hausenblas, 2016, para. 2).

Social marking. Social marking is cognitive process where one side is qualified as natural, thus causing the other side to be depicted as unnatural (Brekhus, 1996, p. 497). In the context of body sizes, all sizes that closely resemble the ideal size are unmarked, and all sizes outside of that group are marked.

Marked. Marked refers to a marked category in social marking; this category is unneutral and often refers to the unnatural. It may imply something about the object and represents a "specialized subset of the whole" (Brekhus, 1996, p. 497, 500; Reber, Allen, $\&$ Reber, 2009). In the context of body sizes, all sizes that do not closely resemble the ideal size are a subset and therefore marked. Petite and plus sizes are examples of marked categories.

Unmarked. Unmarked refers to an unmarked category in social marking; this category is neutral and represents the "whole dimension" or the "nonspecialized whole" (Brekhus, 1996, p. 497, 500; Reber et al., 2009). In the context of body sizes, all sizes that closely resemble the ideal size are unmarked as they are often considered normal or standard. In stores, small, medium, and large sizes, accounting for the various size conversions such as European sizes, are sizes that closely resemble the ideal size and thus unmarked.

Plus-size. "Denoting or relating to clothes of a size larger than those found in standard ranges" (Plus-size, 2017, para. 1).

\section{Literature Review}

Starting with an overview of the overweight and obese rates in Greece, this literature review then discusses sizing discrepancies, social marking, and body positive communication.

\section{Rise of Obesity in Greece and Europe}

In 2013, Greece's population was approximately 11,359,000 (World Health Organization, 2013, p. 1). During this time, 47.9\% of Greece's female population was overweight and 19.9\% was obese (World Health Organization, 2013, p. 1). The World 


\section{A. Camarneiro}

Health Organization (2013) forecasts that the number of obese women in Greece will rise to $40 \%$ by 2030 (p. 1).

The World Health Organization (2013) report also estimated that in 2008 " $46.6 \%$ of [Greece's] population aged 15 years and over were insufficiently active" with $49.4 \%$ of the total being women (p. 3).

As for adolescents, World Health Organization (2013) referenced a study that found that, in 2009/2010, 24\% of 11-year-old girls were overweight and 19\% of 13-yearold girls and $14 \%$ of 15 -year-old girls were overweight (“Adolescents", para. 1).

\section{Clothing Sizes}

Clothing sizes differ substantially between stores. A review of H\&M's size guide in Figure 1 shows that large is a size in between a 44 EUR and a 46 EUR, which is equivalent to a 14 US and a 16 US while Forever 21's large is a size 42 EUR or a 10 to 12 US (H\&M, n.d.; Forever 21, 2017).

\begin{tabular}{|c|c|c|c|c|c|c|c|c|c|c|c|c|c|c|c|c|}
\hline \multirow[b]{2}{*}{ EUR } & \multicolumn{2}{|c|}{ XS } & \multicolumn{2}{|c|}{ s } & \multicolumn{2}{|c|}{ M } & \multicolumn{2}{|c|}{ L } & \multicolumn{2}{|c|}{$X \mathbf{L}$} & \multicolumn{2}{|c|}{$2 X \mathrm{LL}$} & \multicolumn{2}{|c|}{$3 \mathrm{XL}^{*}$} & \multicolumn{2}{|c|}{$4 \mathrm{XL}^{*}$} \\
\hline & 32 & 34 & 36 & 38 & 40 & 42 & 44 & 46 & 48 & 50 & 52 & 54 & 56 & 58 & 60 & 62 \\
\hline US, CA & 2 & 4 & 6 & 8 & 10 & 12 & 14 & 16 & 18 & 20 & 22 & 24 & 26 & 28 & 30 & 32 \\
\hline Bust (cm) & 76 & 80 & 84 & 88 & 92 & 96 & 100 & 104 & 110 & 116 & 122 & 128 & 134 & 140 & 146 & 152 \\
\hline Bust (inch) & 30 & $311 / 2$ & 33 & $34 \% / 2$ & $361 / 4$ & $373 / 4$ & $391 / 2$ & 41 & $31 / 4$ & $451 / 2$ & 48 & $501 / 2$ & $52^{3 / 4}$ & 55 & $571 / 2$ & $593 / 4$ \\
\hline Waist (cm) & 60 & 64 & 68 & 72 & 76 & 80 & 84 & 88 & 94 & 100 & 106 & 112 & 118 & 124 & 130 & 136 \\
\hline Waist (inch) & $231 / 2$ & $25 \% / 4$ & $263 / 4$ & $281 / 2$ & 30 & $31 \frac{1}{2}$ & 33 & $341 / 2$ & 37 & $391 / 2$ & $413 / 4$ & 44 & $461 / 2$ & $48^{3 / 4}$ & 51 & $531 / 2$ \\
\hline Seat $(\mathrm{cm})$ & 84 & 88 & 92 & 96 & 100 & 104 & 108 & 112 & 117 & 122 & 127 & 132 & 137 & 142 & 147 & 152 \\
\hline Seat (inch) & 33 & $341 / 2$ & $361 / 4$ & $373 / 4$ & $391 / 2$ & 41 & $42^{1 / 2}$ & 44 & 46 & 48 & 50 & 52 & 54 & 56 & $573 / 4$ & $593 / 4$ \\
\hline Inseam (cm) & 79 & 79 & 79 & 79 & 79 & 79 & 79 & 79 & 79 & 79 & 79 & 79 & 79 & 79 & & \\
\hline Inseam (inch) & 31 & 31 & 31 & 31 & 31 & 31 & 31 & 31 & 31 & 31 & 31 & 31 & 31 & 31 & & \\
\hline
\end{tabular}

Figure 1. H\&M's size guide for tops, blouses, dresses, and skirts for regular and plus sizes (H\&M, n.d.). 


\section{A. Camarneiro}

\begin{tabular}{|c|c|c|c|c|}
\hline SIZE & xs & $s$ & M & L \\
\hline US/Canada & 0 & $2 / 4$ & $6 / 8$ & $10 / 12$ \\
\hline UK & 6 & $8 / 10$ & 12 & 14 \\
\hline EU & 34 & $36 / 38$ & 40 & 42 \\
\hline EU Italy & 38 & $40 / 42$ & 44 & 46 \\
\hline Bust (IN) & 32 & $34-35$ & $36-37$ & $38-39$ \\
\hline Bust (CM) & 81 & $86-89$ & $91-94$ & $96-99$ \\
\hline Waist (IN) & $24-25$ & $26-27$ & $28-29$ & $30-31$ \\
\hline Waist (CM) & $61-64$ & $66-69$ & $71-74$ & $76-79$ \\
\hline Hips (IN) & $33-34$ & $35-36$ & $38-40$ & $42-44$ \\
\hline Hips (CM) & $84-86$ & $89-92$ & $96-102$ & $107-112$ \\
\hline SIZE & $0 \mathrm{x}$ & $1 \mathrm{X}$ & $2 \mathrm{x}$ & $3 x$ \\
\hline UK & 18 & 20 & 22 & 24 \\
\hline EU & 46 & 48 & 50 & 52 \\
\hline EU Italy & 48 & 50 & 52 & 56 \\
\hline Bust (in) & $42-43$ & $44-45$ & $46-47$ & $48-50$ \\
\hline Bust $(\mathrm{cm})$ & $107-109$ & $112-114$ & $\underline{117-120}$ & $122-127$ \\
\hline Waist (in) & $35-36$ & $37-38$ & $39-40$ & $41-43$ \\
\hline Waist $(\mathrm{cm})$ & $89-92$ & $94-97$ & $99-102$ & $104-109$ \\
\hline Hips (in) & $45.5-46.5$ & $47.5-48.5$ & $49.5-50.5$ & $51.5-53.5$ \\
\hline Hips $(\mathrm{cm})$ & $116-118$ & $121-123$ & $126-128$ & 131 - 136 \\
\hline Thigh (in) & 27 & 28 & 29 & 30.5 \\
\hline Thigh $(\mathrm{cm})$ & 69 & 71 & 74 & 77 \\
\hline
\end{tabular}

Figure 2. Forever 21's size guide for apparel for regular and plus sizes (Forever 21, 2017).

Figure 1 and Figure 2 show the inconsitencies between stores regarding sizing. The two figures also show how some stores classify size 44 to 46 EUR or 16 US as large and others as plus size. The waist size in inches for a large size also differs between H\&M and Forever 21. Labelling theory emphasizes why the terminology used can have an impact on how a person behaves (Murray et al., 2011, p. 176), which may mean the term plus size might alter how a person manages their food consumption and physical activity. This is because if a person is in the presence of a distinct, size-discriminatory label, they may begin to self-identify with that label and start behaving in a consistent manner (Murray et al., 2011, p. 176). In other words, if a person is continuously called plus size or simply fat, they may consume food like a stereotypical "fat" person would.

The average clothing size is also increasing (Tali, 2016, para. 1). While the previous average clothing size for women was thought to be a size 14 US, it was recently discovered that this number has increased, and the average size is actually a size 16 US to 18 US (Tali, 


\section{A. Camarneiro}

2016, para. 1). Regardless of this increase in the average size, many clothing retailers still deem size 16 US and above to be plus size clothing (Tali, 2016, para. 3).

\section{The Ideal Size}

There is an ideal body size- the socially constructed bodily appearance deemed to be most beautiful — that varies between countries (Aronson et al., 2013, pp. 214-215). This ideal body size is often held as the standard for women to achieve; it is pervasive in societies and maintained through media, culture, and social interactions (pp. 214-215). Individuals are taught how to appear appropriate through informational social influence while normative social influence persuades individuals to comply with what's socially acceptable. Moreover, imposing an ideal size is problematic for several reasons, explored below.

For instance, the ideal body size has not been consistent between cultures and societies or across time (Aronson et al., 2013, p. 215). Anderson et al. (as cited in Aronson et al., 2013) studied 54 cultures to decipher what various cultures considered to be the ideal female form (p. 215). In each society, data on the reliability of food supply was collected. Researchers discovered that societies with low food supply, individuals with heavier bodies were described as beautiful; an assumption was made that this was because "women who have enough to eat... [are] healthy and fertile" (p. 215). In other words, cultures found to have unreliable food supplies preferred heavy to moderate sized women. Researchers also discovered that "heavy to moderate [female] bodies were preferred by the vast majority in all cultures" with the exception being North America, where thinness was preferred (p. 215).

Equally interesting is that even in one culture, Western culture for example, the ideal body size has not been consistent across time (Aronson et al., 2013, p. 215). Photograph analyses in magazines like Vogue and Ladies' Home Journal show a dramatic change in the Western ideal body size during the beginning of the twentieth century: prior to the 1920s, a more voluptuous female form was glorified and sexualized, and, following the 1920s, "the preferred look for women was rail-thin and flat-chested" (p. 215). Another change occurred in the 1940s when the curvaceous pin-up girl became the ideal body size; this was maintained in the 1950s and exemplified by Marilyn Monroe. In the 1960s, the thin silhouette once again became popular and has, in fact, become an even stronger reinforced standard today (Aronson et al., 2013, p. 215).

Overall, this information reinforces the idea that beauty, specifically body size, is subjective and demonstrates that women may feel socially influenced to conform to a 


\section{A. Camarneiro}

certain, possibly unattainable, body size even though that size has been proven to be inconsistent culturally and generationally.

\section{Social Marking}

Creating various subsets through unmarked and marked categories can cause individuals within that marked or subset group to feel abnormal and create the perception of a social divide (Brekhus, 1996, p. 497, 500). The researcher noted that a mark can be implied or literal. An implied marking could be something such as a tone or fact that implies it is made for or excludes the subset. A literal marking in this research study could be something such as a plus-size section in a clothing store, the word plus on a clothing tag, or another similar item that literally labels or "marks" the wearer as being part of a subset group. The danger of social marking is when a person assigns stereotypical information to the entire subset group (Brekhus, 1996, p. 497).

\section{Discrimination of Overweight and Obese Persons}

Discrimination of plus size persons is ongoing. "Weight stigma is more pervasive and intense than racism, sexism, and other forms of bias" (Brochu \& Esses, as cited in Tomiyama \& Mann, 2013, p. 4). Size-discrimination is one of the last, legal and acceptable forms of discrimination in America (Tomiyama \& Mann, 2013, p. 4; Puhl \& Brownell, 2001, p. 788). Education, employment, and health care are three vital "areas of living... [where] clear and consistent stigmatization, and in some cases discrimination, can be documented" (Puhl \& Brownell, 2001, p. 788). Puhl and Brownell (2001) noted 24\% of nurses are "repulsed by obese persons" (p. 788). In addition, in a survey study by Tomiyama and Mann (2013), the majority of participants of the survey held negative views of obese persons (p. 4).

It is not difficult to see discrimination of overweight or obese persons in other areas such as social media; in fact, Figures 3, 4, 5, and 6 are all comments on various body positive videos on Facebook that demonstrate a member of the general public's negative or stereotypical views of overweight or obese persons. Moreover, even posts regarding overweight or obese children expressing body positivity contain body shaming comments; this can be seen in Figures 4, 5, and 6. 
How can someone get THAT big in the first place? I always wonder when I see fat people out there. It doesn't happen overnight! People don't notice their size are getting out of control? But kuddos for this

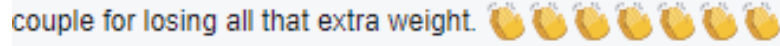

Like - Reply - OPS $37 \cdot 21$ hrs

$\rightarrow 15$ Replies $1 \mathrm{hr}$

Figure 3. A Facebook comment on a couple's weight loss story (2017, Facebook comment).

I love body positivity. But, please, can we stop glorifying obesity? It is UNHEALTHY. I understand some people gain weight for health reasons, but being overweight is not something that should be widely accepted. It can kill people.

Like - Reply - OPS 368 - June 9 at 9:29am

Figure 4. A Facebook comment on a video about a young girl who created a body-positive fashion line in response to being bullied. This post also has a high number of social reactions - from love, like, to hate-that pushes this post to the top of the comments, thereby making it more visible to those who read the comments under the video (2017, Facebook comment).

The thing is, it doesn't look graceful or smooth when a heavyweight person does it. she can dance, sure. But it doesn't look as good as it could.

Like - Reply - 983 - August 16 at $1: 34 \mathrm{pm}$

$\rightarrow 16$ Replies

Figure 5. A Facebook comment posted on a video about a young body positive dancer who was striving to break stereotypes about the appropriate body size for a dancer (2017, Facebook comment).

No hate but like it's not healthy to be that overweight at her age and I understand if it's because of a medical problem but if she's able to do sit about it she shouldn't be preaching to everyone saying that's ok but I mean live ur truth

Like - Reply - (1) 3 - August 17 at 7:14am

$\rightarrow 2$ Replies

Figure 6. A Facebook comment posted on a video about a young body positive dancer who was striving to break stereotypes about the appropriate body size for a dancer (2017, Facebook comment).

These counterintuitive comments demonstrate that some individuals do not understand the impact of body shaming. It also demonstrates that even videos 
encouraging healthy consumption, mindset, or activity are subject to condescending, sizediscriminatory comments solely due to the person's size.

Self-discrimination regarding body weight also occurs. In the survey study by Tomiyama and Mann (2013), 88\% of participants indicated they were unhappy with their appearance, which ultimately suggested that individuals were unhappy with their weight (p. 4). Despite some critics arguing that shaming reduces obesity, size-discrimination is proven to negatively impact mind and body health (Callahan, as cited in Mann et al., 2015, p. 4; Tomiyama \& Mann, 2013, p. 4).

Body positive communication is required to deconstruct negative and unhelpful views regarding obesity and develop a more inclusive society to create a positive impact on mind and body health overall.

\section{Negative Body Communication}

In response to a dramatic increase in obesity levels in the past 35 years, various health scholars have presented weight-reduction strategies (Mann et al., 2015, p. 706; Tomiyama \& Mann, 2013, p. 4). The most notable was proposed by Callahan (as cited in Mann et al., 2015; Tomiyama \& Mann, 2013) who suggested societies use stigma to dissuade individuals from being obese, which is a plan that is unfortunately "well underway" (p. 707; p. 4).

In a survey conducted by Callahan (as cited in Tomiyama \& Mann, 2013), it was concluded that "overweight and obese people do not find anything problematic aboutor even notice-their weight" (p. 4). However, in a survey conducted by Rand and Macgregor (as cited in Tomiyama \& Mann, 2013) on gastric bypass patients who had lost more than 100 pounds, it was discovered that most of those individuals "would rather be deaf, blind, have heart disease, or lose a leg than gain back the weight they lost"; all of the individuals indicated "they would give up being a multimillionaire to be normal weight" (p. 4).

What is more, weight stigma "causes increased eating, predicts exercise avoidance, [and] depletes the very mental resources needed to control one's behavior"; it is also "linked to an increased (not decreased) risk of becoming obese over time" (Hunger \& Tomiyama, 2014, as cited in Mann et al., 2015, pp. 707-708).

In a study by Tomiyama and Mann (2013), researchers "conducted a small randomized experiment by asking 372 individuals... six [weight-stigmatizing] questions or [six] neutral questions about ecofriendly behaviours" (p. 4). Following the experiment, the 
researchers offered food and asked individuals to select "any and all foods they would like to eat at that moment" (p. 4). The individuals who answered the weight-stigmatizing questions selected "statistically significantly higher amount of sugary foods... [and] significantly more calories... than those who answered neutral questions" (Tomiyama \& Mann, 2013, p. 4).

Researchers add that campaigns that use weight stigma will more than likely fail at achieving an overall reduction in obesity (Mann et al., 2015, pp. 707-708). Engeln (2017) documented an analysis of "fitspiration websites" and noted that nearly all of these sites closely resembled "openly pro-anorexia sites" by both being filled with weightdiscriminatory communication, promotions of unhealthy eating, and comments that connect food to guilt (para. 4). These sites and type of communication focuses fitness on being something a person sees opposed to something a person feels or can do (Engeln, 2017, para. 3).

In another example, after a group of 400 women, all of whom took fitness classes, were surveyed, it was discovered that most women hated hearing body-shaming comments during their workout from fitness instructors; in fact, $56 \%$ of the individuals surveyed listed appearance-related comments as "their 'least favourite' motivational comment" (Engeln, 2017, para. 5).

In short, "body shame is the enemy of a healthy, long-term approach to taking care of your body" (Engeln, 2017, para. 4), and this information supports the use of body positive communication over negative body communication to encourage others to maintain a healthy mind and body.

Further, paradoxical methods to reducing obesity are promoted by medical professionals. One of the most common strategies prescribed by medical professionals for weight loss — restrictive dieting — can in fact cause weight gain (Mann et al., 2015, p. 706). This occurs through "deprivation [that] ultimately triggers a biological starvation response... [wherein] neurological changes render food especially attention-grabbing... and more rewarding to consume" (Adam \& Epel, as cited in Mann et al., 2015, p. 706). It is also said that "general self-control, ability, or willpower, is reduced... or surprisingly fragile" (Page et al., as cited in Mann et al., 2015) and that weight short term weight loss often cannot be maintained long term (Franz et al.; Tomiyama, Ahlstrom, \& Mann, as cited in Mann et al., 2015, p. 706).

Visual communication, specifically the visual representation of the ideal body size, is known to impact women and their self-esteem. In findings coined by researchers as 
"Victoria's Dirty Secret," it is proven that women exposed to attractive, thin models in advertisements report greater body dissatisfaction and are more inclined to use their appearance as the basis of their self-esteem (Aronson et al., 2013, pp. 216-217).

Further, to be liked and accepted, i.e. through normative social influence, women "attempt to create the ideal body through dieting and, more disturbingly, through eating disorders such as anorexia nervosa and bulimia" (Aronson et al., 2013, p. 217). It is estimated that nearly 80 percent of young women want to be skinnier and that roughly 250,000 of Canadian women ranging from 13 to 40 have anorexia nervosa and "twice as many have bulimia” (p. 217). Ten to $15 \%$ of these illnesses are fatal (p. 217).

\section{Body Positive Movement and Communication}

In recent years, a movement called the body positive movement has emerged in response to body dissatisfaction and unrealistic beauty ideals (Cwynar-Horta, 2016, p. 37; Schreiber \& Hausenblas, 2016, para. 1). Despite this movement growing over the past decade, academic studies relating to the body positivity movement are lacking, and a definition for the body positivity movement varies depending on the source (CwynarHorta, 2016, p. 37; Schreiber \& Hausenblas, 2016, "How do you”, para 1-3).

Cwynar-Horta (2016) indicated that the main goal of the movement was to "address unrealistic ideals about beauty, promote self-acceptance, and build self esteem" (p. 37). Dunn, as cited in Schreiber \& Hausenblas (2016) also added that this movement encourages self-love and that a person's appearance does not define their self-worth ("How do you", para. 1).

In all the definitions, several themes emerged regarding self-love, particularly self, body-, and flaw-acceptance. It is important to note that the body positivity's movement key message does not condemn self-improvement or embrace unhealthy eating: it simply indicates that self-worth, love, and respect should not be dependent on body weight, size, or shape.

This movement's presence can be found in advertisements in the last decade, such as Dove, Aerie, and Old Navy, which have included body positive messaging and inclusive imagery; these advertisements showcase various sizes of bodies with the assumed aim of communicating that that company accepts every body and does not discriminate based on size (Dubecky, n.d. para. 2, 6-7, 9; Schreiber \& Hausenblas, 2016, para. 2).

Since 2012, the presence of body positive messages on Instagram has also increased, and, in 2013 when Instagram became an advertising platform, even more plus- 
sized campaigns were seen as a result (Cwynar-Horta, 2016, pp. 37-38). News outlets have contributed to body positive discourse by circulating stories about individuals' "experiences with eating disorders", challenging the dominant ideal body size, or replying to "body shamers" (pp. 37-38).

However, there is also some backlash to the movement and its key message. Commenters on Facebook, as seen in Figure 4 and Figure 6, and news articles question whether body positivity is "too positive" about obesity by glorifying unhealthy habits and deeming these habits acceptable (Fraser, 2017, para. 1). However, the need for body positive communication is made clear when analyzing the effects of body shaming. To add, "misconceptions about obesity have hindered policy efforts to promote health" (Mann et al., 2015, p. 706). The body positivity movement is therefore required to raise awareness and in turn promote health.

\section{Methodology}

The researcher collected qualitative data; this means that the researcher collected empirical data, such as information pertaining to attributes, to gain an understanding of the research question (Wimmer \& Dominick, 2014, p. 48).

The researcher used unobtrusive observation to collect data. Unobtrusive observation comes from combining "unobtrusive measurement" and "observation"; unobtrusive measurement means the subject is unaware data is being collected, reactivity, the reaction from a subject, is eliminated, and the researcher is recording "naturally occurring phenomena relevant to the research task" (Wimmer \& Dominick, 2014, p. 130).

Systemic random sampling was used to photograph images and collect data on clothing store sizes. Systemic random sampling means "every nth subject, unit, or element is selected in the population” (Wimmer \& Dominick, 2014, p. 97).

Data were collected during the week of May 8 to May 15, 2017 in downtown Athens, Greece. The research sample consisted of women, female mannequins, and women's clothing only.

For imagery, the researcher walked through populated shopping districts and in the downtown area of Athens, Greece and photographed every billboard, front-of-store imagery, and wall imagery containing women, as well as female mannequins. When analyzing the imagery that contained multiple people (both male and female), the researcher used systemic random sampling by specifically studying the first two females 
found on the left of the image. In other words, every item seen during collection was photographed and every person after the first was analyzed.

A data sheet was prepared to analyze photographs. The data sheet contained the size of the women or female mannequins pictured, a short description of the clothing worn, including tone and whether the persons or figures shown were part of the marked or unmarked category, and additional observations. The size and social marking sections contained a "not enough data" option. Thirty-two images were reviewed; however, one image contained both mannequins and a photograph of a woman, so 33 data sheets were created for 33 subjects.

For clothing size selection, every fifth item was selected for analysis. The researcher would enter the store and turn left, and, moving left to right, select the fifth item on the clothing rack. The researcher would then record all the sizes available for each piece of clothing in that style, and move on to the next fifth item. In some instances, the researcher was required to select a rack due to sporadic store layouts or move to a new rack to avoid communication with sales associates. To record sizes, the notepad application on the researcher's iPhone was used. This was done to avoid attention and thus communication with sales associates, security guards, or customers. In total, 15 stores were entered and analyzed.

\section{Results}

\section{Images}

Out of the 33 images, $22(66.7 \%)$ were mannequins and $11(33.3 \%)$ were photographs of models. This includes the one image in both categories. The women were sized small, medium, large, extra large, above extra large, and as not enough data. Wide curves but small waists or large amounts of clothing made some models and mannequins difficult to size. In cases where the woman or figure appeared to be between two sizes, such as small-to-medium or medium-to-large, the data sheet was checked in both categories. 
The sizes recorded are shown in Figures 7 and 8.

\begin{tabular}{|c|c|c|c|c|c|c|}
\hline & Small & Medium & Large & $\begin{array}{l}\text { Extra } \\
\text { Large }\end{array}$ & $\begin{array}{l}\text { Above } \\
\text { Extra } \\
\text { Large }\end{array}$ & $\begin{array}{c}\text { Not } \\
\text { enough } \\
\text { data }\end{array}$ \\
\hline Number of items per size & 44 & 11 & 3 & 0 & 0 & 0 \\
\hline $\begin{array}{l}\text { Percentage of number of } \\
\text { items per size out of the } \\
\text { total, } 60\end{array}$ & $73.3 \%$ & $18.3 \%$ & $5.0 \%$ & $0 \%$ & $0 \%$ & $3.3 \%$ \\
\hline $\begin{array}{c}\text { Number of items paired with } \\
\text { other size }\end{array}$ & 6 & 9 & 3 & 0 & 0 & - \\
\hline $\begin{array}{l}\text { Percentage of number of } \\
\text { items paired with other size } \\
\text { out of the total, } 60\end{array}$ & $10.0 \%$ & $15.0 \%$ & $5.0 \%$ & - & - & - \\
\hline $\begin{array}{c}\text { Percentage of number of } \\
\text { items paired with other size } \\
\text { out of the number of items } \\
\text { per size }\end{array}$ & $13.6 \%$ & $81.8 \%$ & $100 \%$ & - & - & - \\
\hline
\end{tabular}

Figure 7. Sizes recorded for women and mannequins.

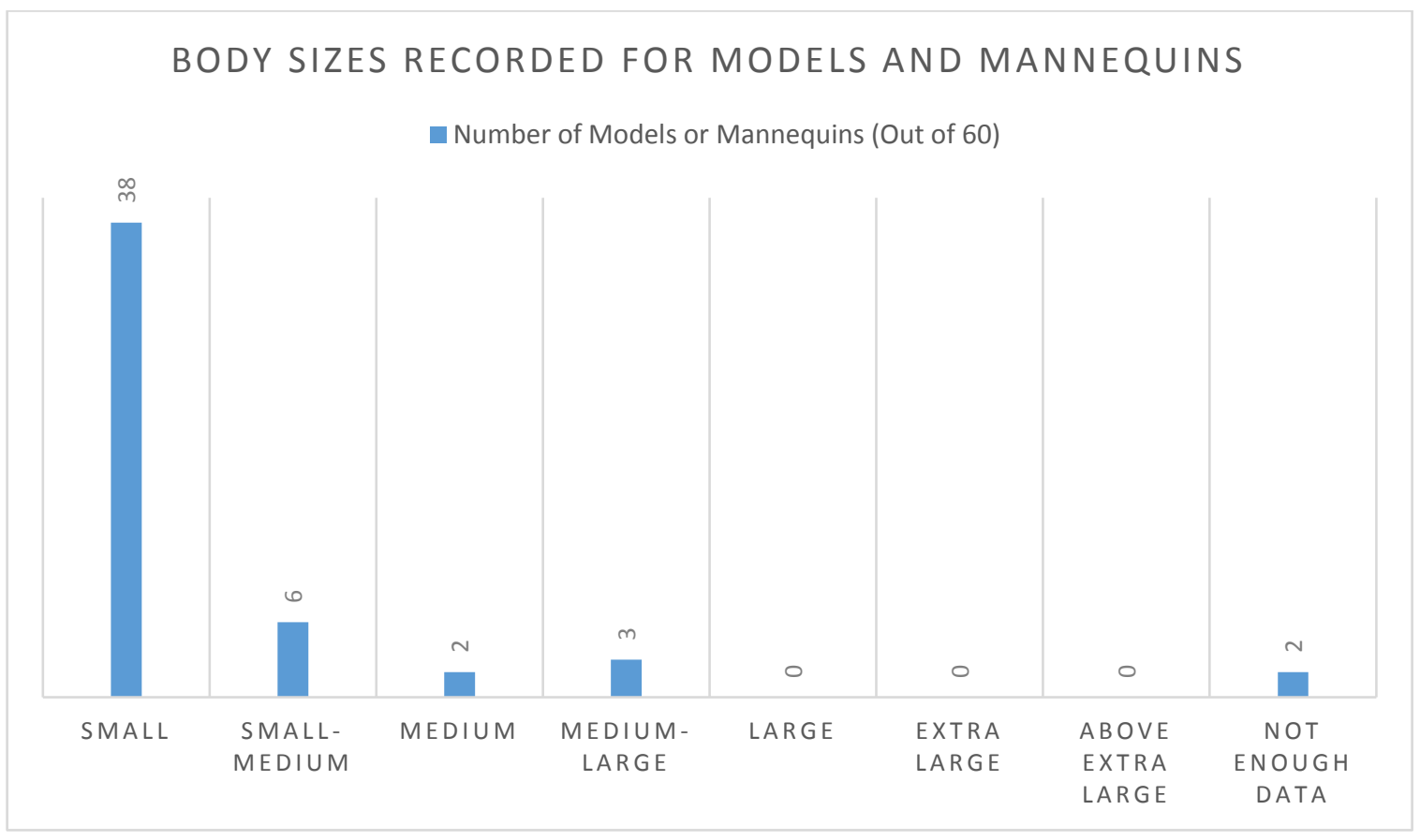


Figure 8. Body sizes recorded for women and figures in a bar graph. This bar graph includes paired sizes as its own category.

The tone of the images was also recorded, and 13 out of the $33(59.0 \%)$ were described as stylish, eight $(24.2 \%)$ as provocative, and five $(15.2 \%)$ as alluring.

Out of the 60 women and figures analyzed, 58 belonged in the unmarked category, two could not be analyzed, and none belonged to the marked category.

\section{Clothing Stores}

The 15 stores analyzed were divided into three groups based on the amount of available clothing on the rack: high selection, moderate selection, and limited selection. High selection stores mainly consisted of international clothing stores. Moderate selection and limited selection stores mainly consisted of European-based or local Athens clothing stores.

The stores were reviewed as follows:

High Selection:

- $\mathrm{H} \& \mathrm{M}$

- Forever 21

- American Eagle

Moderate Selection:

- Pull and Bear Clothing Store

- Admiral Sport Shop

- Lycabette

- Nike

- Tezenis

- The Fashion

Limited Selection:

- Traffico

- Celestino Vice Versa

- Unknown Name (Alternative Clothing Store)

- Kapeo 
18 A. Camarneiro

- Lacoste

- Metropolis Fashion City

Below is an overview of the sizes recorded during data collection. Every size within alpha sizes (small, medium, large, etc.) and numerical European sizes (32 EUR to 48 EUR) were recorded. US sizes, UK sizes, one size items, and sizes determined by inches were included in the unknown section.

In total, 202 alpha sizes were recorded, 66 numerical European sizes were recorded, and 36 unknown sizes were recorded.

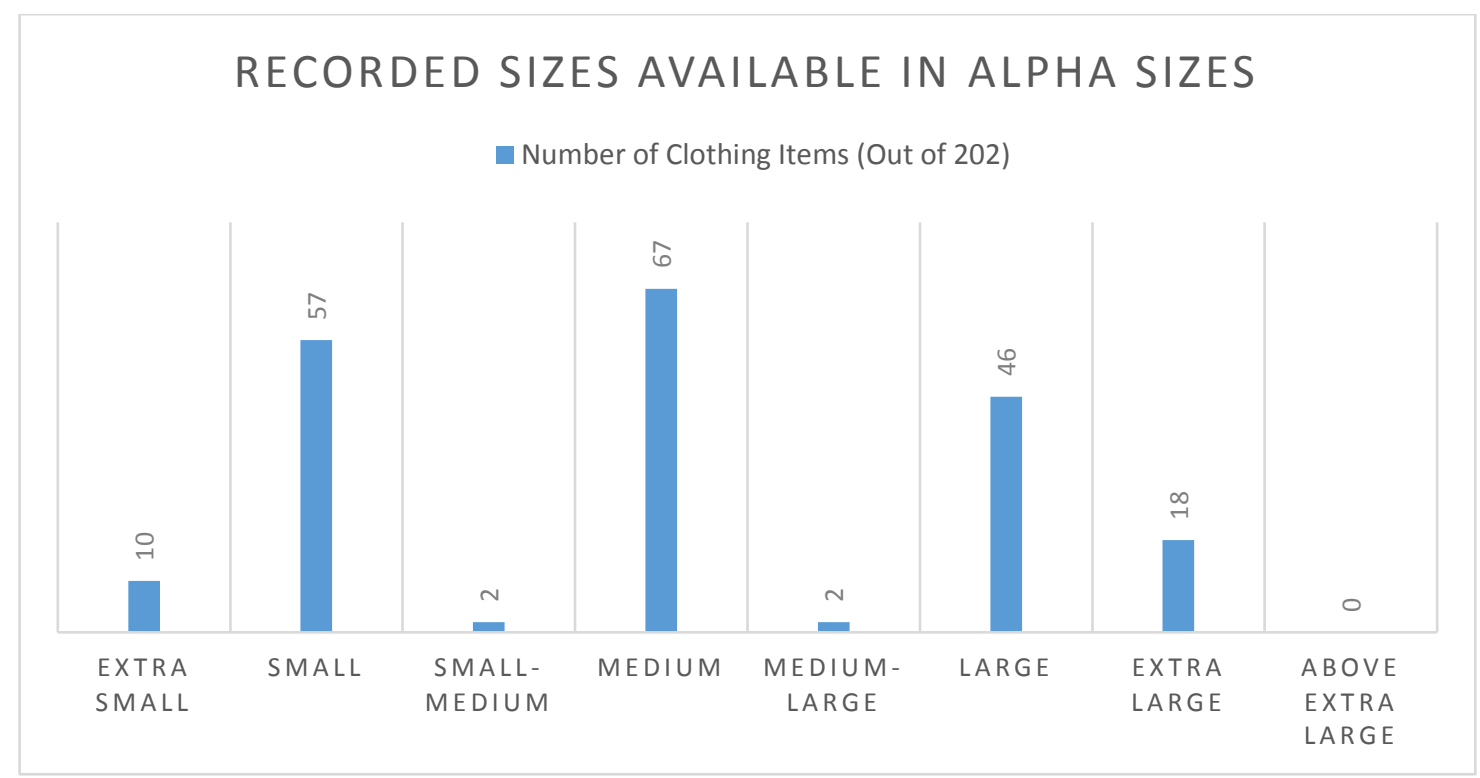

Figure 9. Number of items in each of the alpha size categories that were recorded.

Thus, in the alpha category, medium was the most common size. Medium sizes were nearly four times more common than extra-large sizes. A medium is equivalent to a 40 EUR to 42 EUR according to H\&M's size chart and 40 EUR according to Forever 21's size chart. 
The sizes in the European numerical size category recorded can be seen in Figure 10 below:

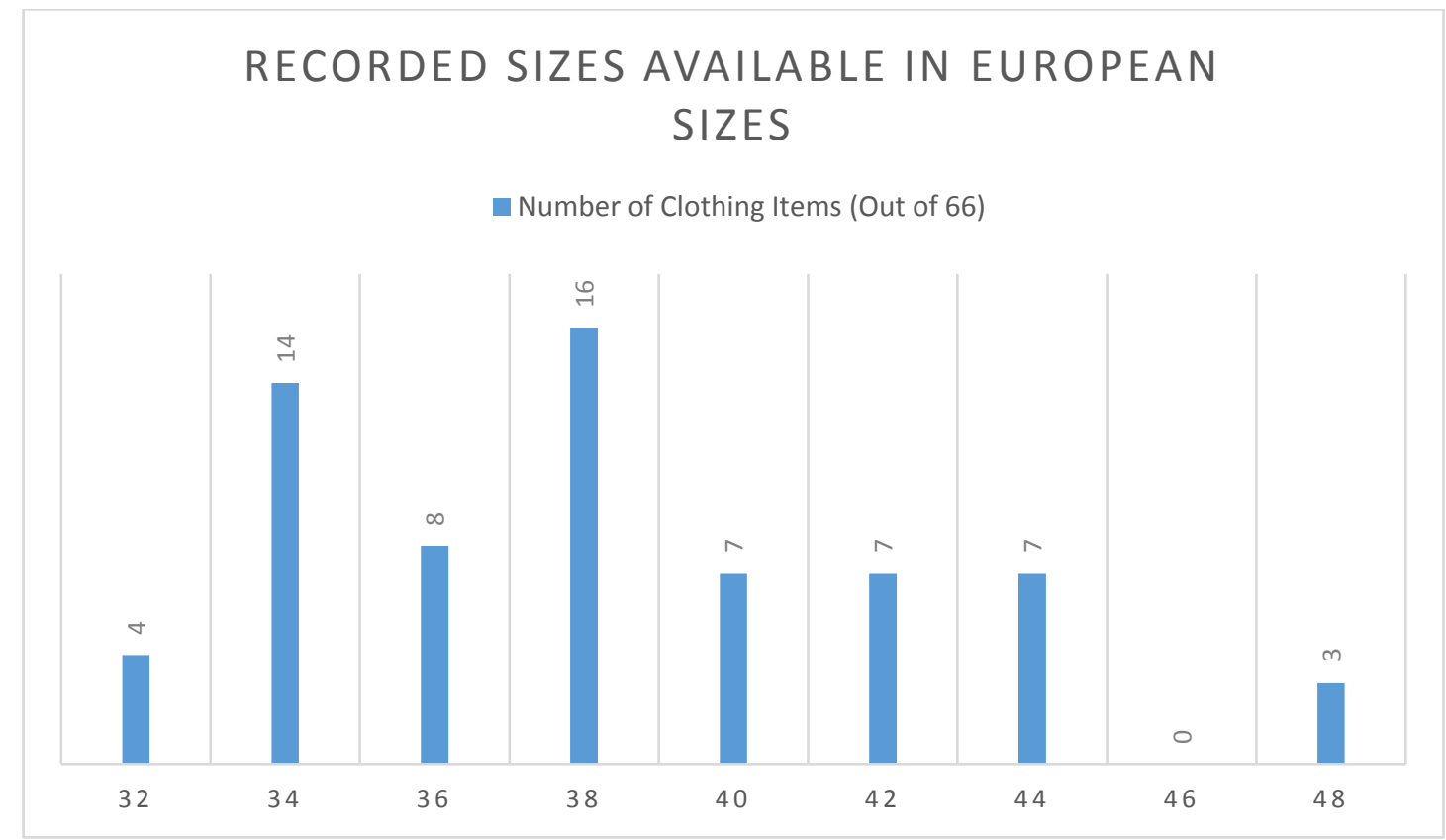

Figure 10. Number of items in each of the European size categories that were recorded.

For European numerical sizing, the most common sizes were 34 EUR and 38 EUR. These are defined as an extra-small and small, respectively, according to both H\&M and Forever 21's size charts.

On the clothing data sheets, used to analyze the 202 alpha sizes, the 66 European numerical sizes, and the 36 unknown sizes, the terms "accurate" or "reasonable" were used to define 17 items, words synonymous to "appearing to fit smaller than the size on the tag" were used 18 times, and words synonymous to "appearing to fit larger than the size on the tag" were used nine times.

There were other observations made during collection. For example, sizes fluctuated heavily with items in different stores and also with items in their own store. In numerous stores, sizes appeared smaller or larger depending on the style or the brand of the clothing, and sizes of certain items were sometimes vastly different than the same size of another similar item. As well, at times, tags indicated the items went to higher sized items, generally extra-large, without any of those larger items being available for purchase. 
Multiple workout pant sections were also recorded. These sections, in H\&M, Nike, and Admiral Sport Shop were recorded in addition to the items above and without using the systematic random sampling system.

In Admiral Sport Shop, 15 workout pants were found on one rack and recorded: one of the 15 pants were large, and two of the 15 pants were extra-large. What's more is those extra-larges appeared to be nearly the same size as a small of another style.

WORKOUT PANTS RECORDED AT ADMIRAL SPORT SHOP

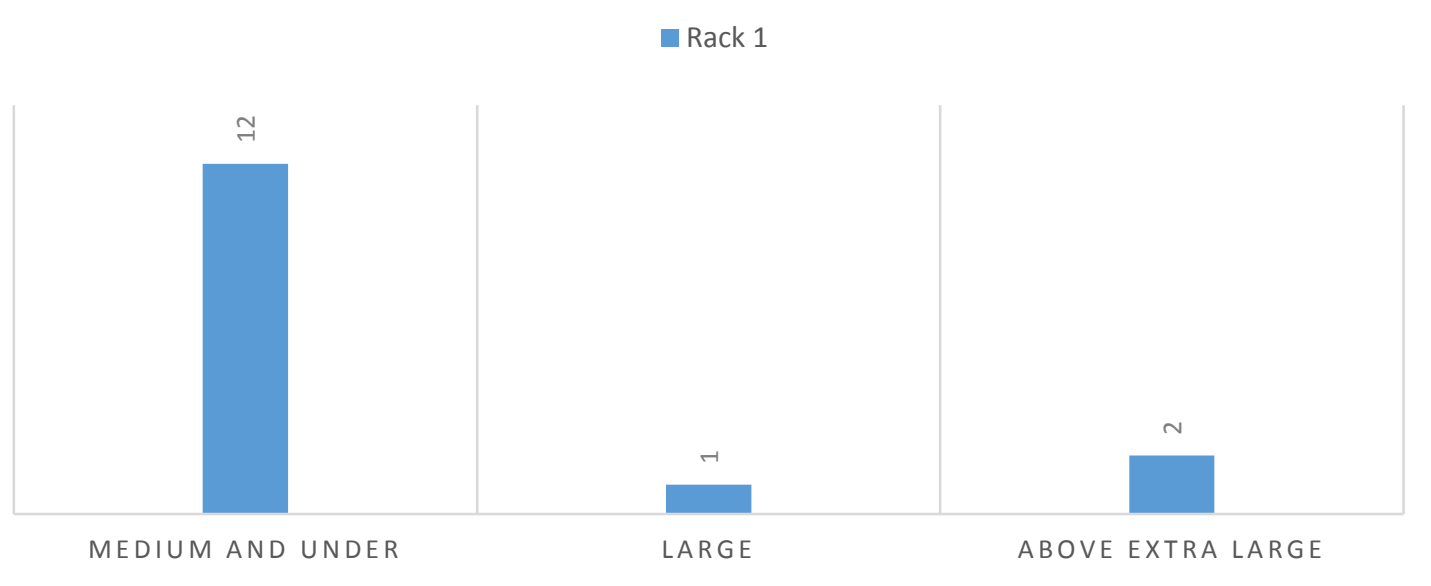

Figure 11. Workout pants at Admiral Sport Short split into "medium and under", "large", and "extra large".

At Nike, there were three racks of workout pants. The first rack had 11 total, and one was large; the second rack had 11 and 4 were large, and the third rack had 9 total and none were large. No extra larges were recorded.

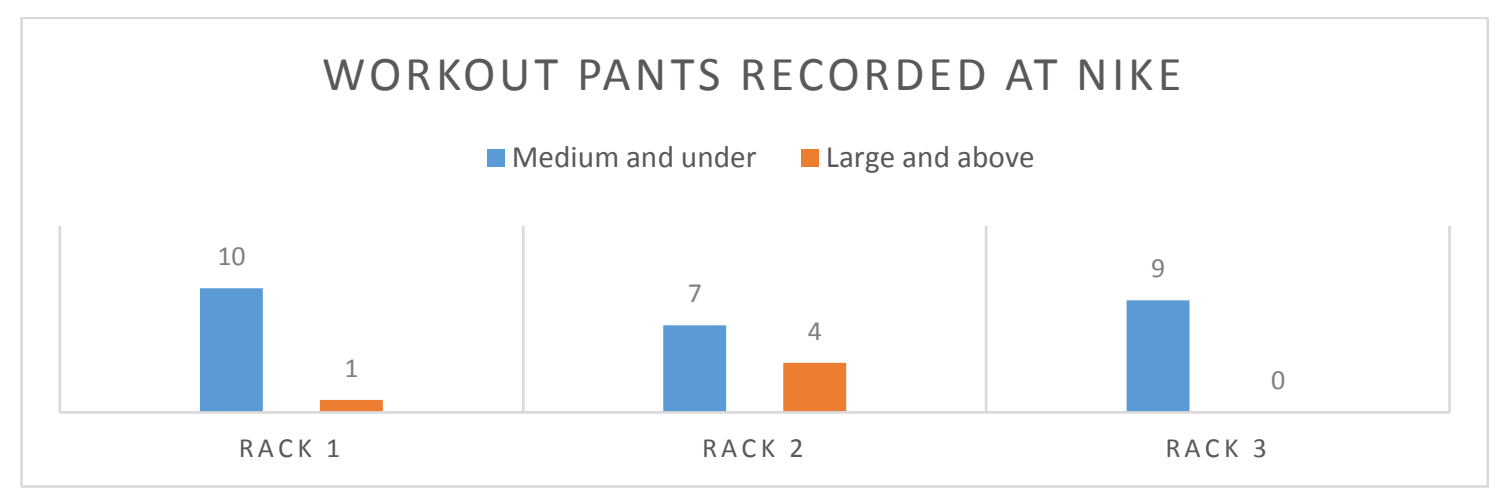

Figure 12. Workout pants at Nike split into "medium and under" and "large and above." 


\section{A. Camarneiro}

H\&M also had a section of workout pants that has a size range of extra-small to large: no extra-larges of this item were manufactured to sell in store.

Also in H\&M, for example, it was noticed that one short pair of denim shorts had smaller size range than other items.

In Forever 21, similar size range limits were noticed. A yellow halter top, for example, included the size range extra-small to large on its tag (Figure 13).
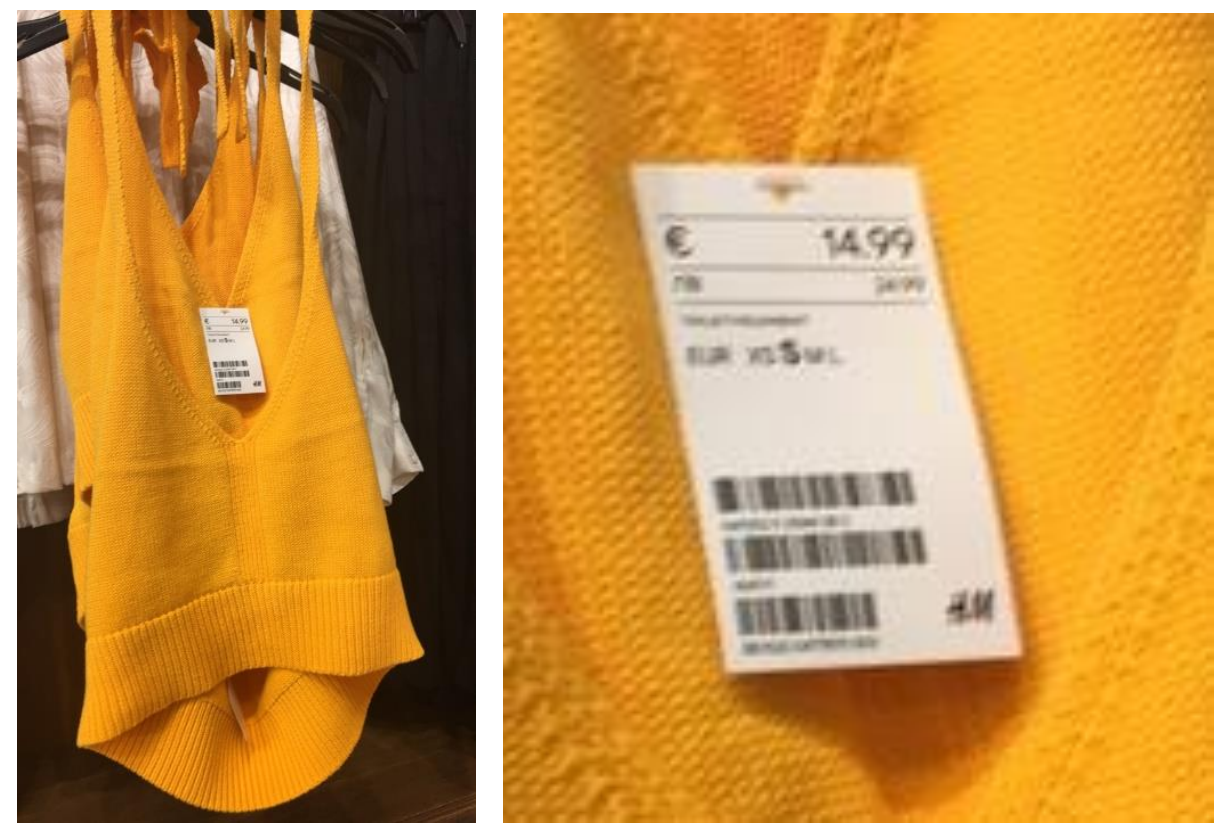

Figure 13. Picture of yellow halter top and picture of the close-up of tag that indicated the size range of extra small to large, inclusive

Forever 21 also had a plus size section in store. The items in the plus size section were not included in the sizes data because plus size items are directed to a subset group, not a "normal" group. In other words, the researcher aimed to collect data in stores advertised for everyone opposed to those advertised to a select group.

In this section, an image of a "plus size" woman hung on the wall wearing a crop top. Her clothes made it difficult to determine her actual size, i.e. if she was plus size or not. It was also noticed that the crop top clothing item in the image was not found in the plus size section for purchase by customers. 
This image was also not included in the images of women and mannequins because it is from a subset section and is not an advertisement directed at what the store defines as "normal".

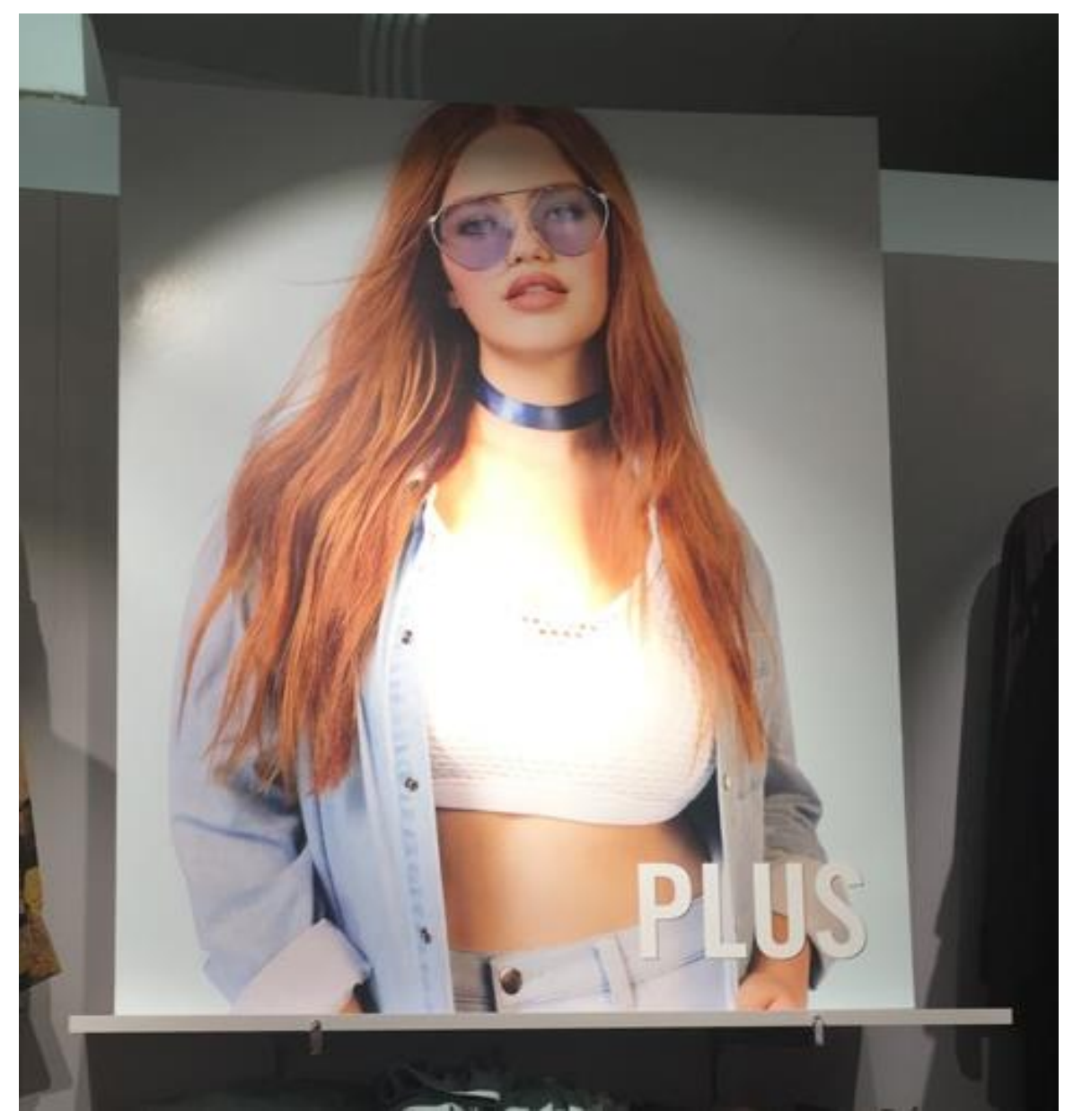

Figure 14. Image of plus size model in photograph hanging in plus size section of Forever 21. 
Moreover, there was only one shirt in the plus size section that showed part of midriff, and it was a crop top with tassels attached to the bottom. The tassels covered some of the midriff.

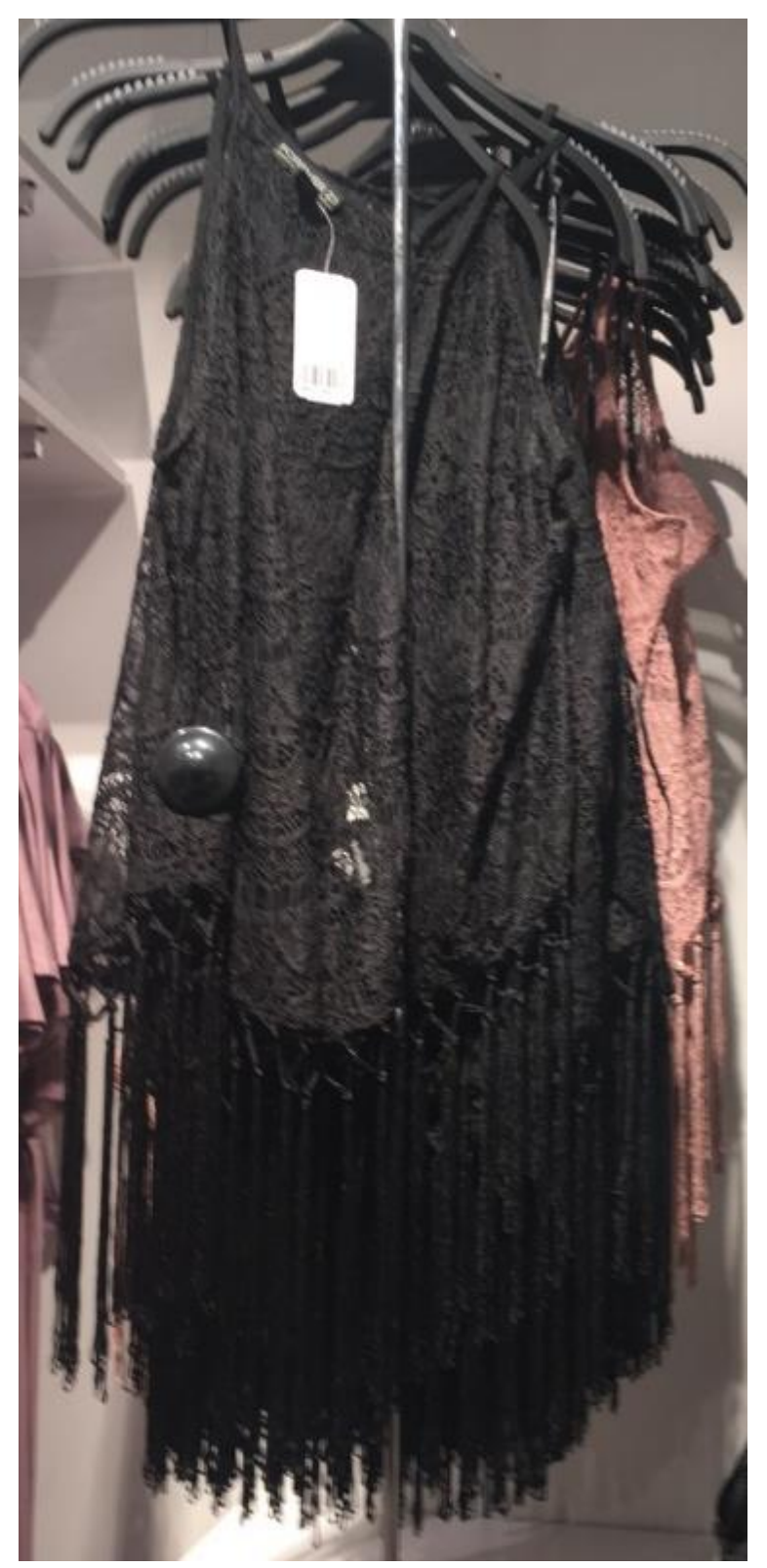

Figure 15. Image of crop top with tassels located in Forever 21's plus size section. 


\section{A. Camarneiro}

Shorts were also different in the plus size section. Throughout of rest of the store, numerous short denim shorts were available for purchased by customers. In the plus size section, however, there were no short denim shorts available for purchase. The shorts that were available to plus size women were either baggy or spandex and all appeared to be for working out or lounging at home. Figure 16, for instance, shows baggy shorts found in the plus size section, and Figure 17 shows two pairs of tight-fitted denim shorts in the "normal" section of the store.

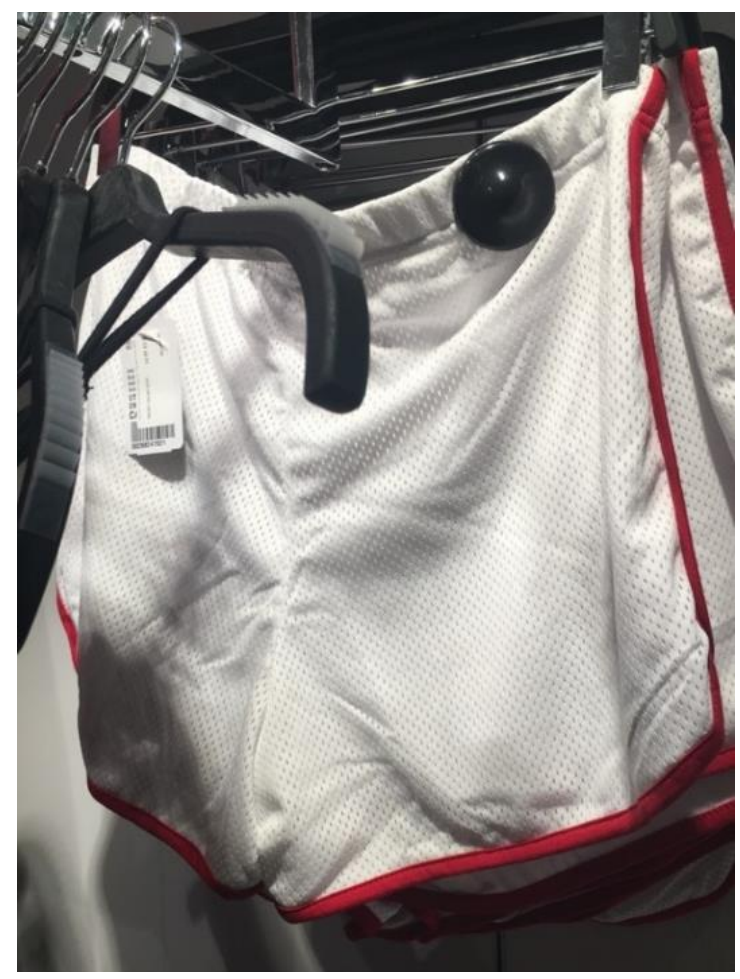

Figure 16. Image of short baggy shorts in plus size section. 


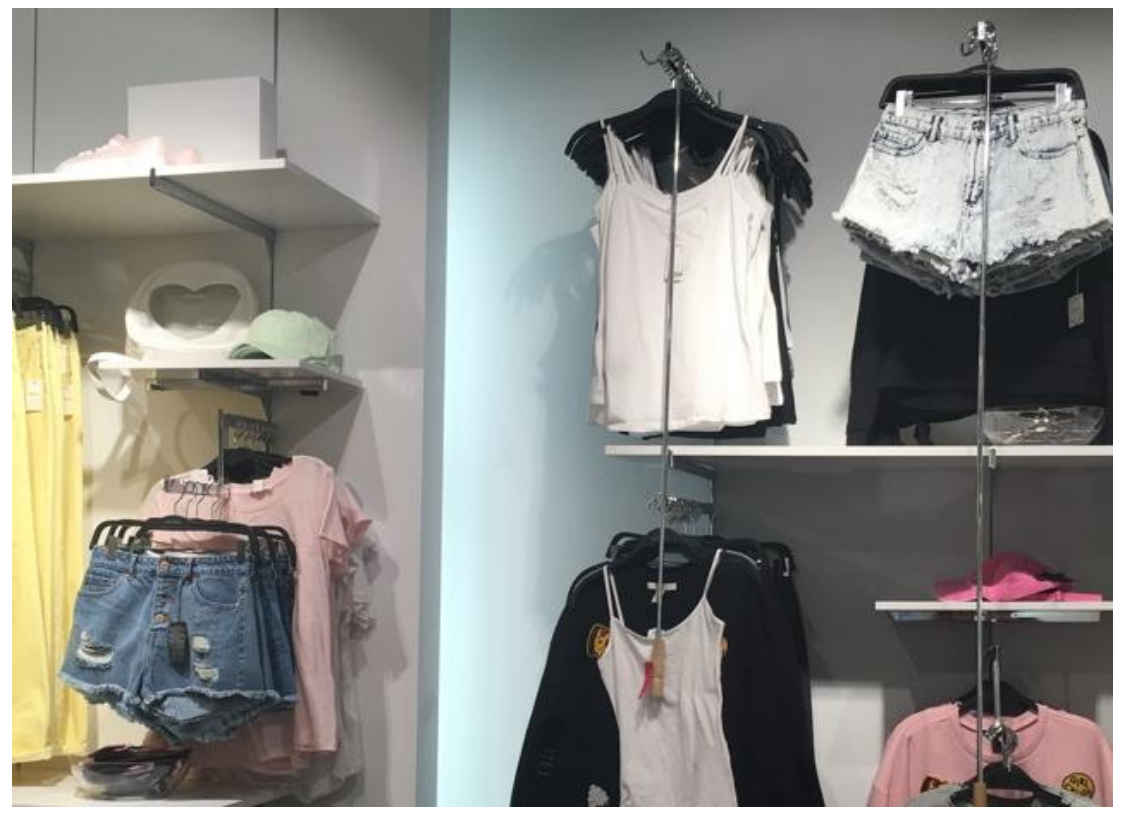

Figure 17. Image of two short denim shorts in the 'regular' section.

In the four-storey Forever 21 store, the plus size section was in the far corner of the top floor. It was also located next to a workout section that did not include plus size items. Further, as you can see from Figure 18, the clothing in this section contained a literal marking that set the clothing apart from 'normal' sizes.

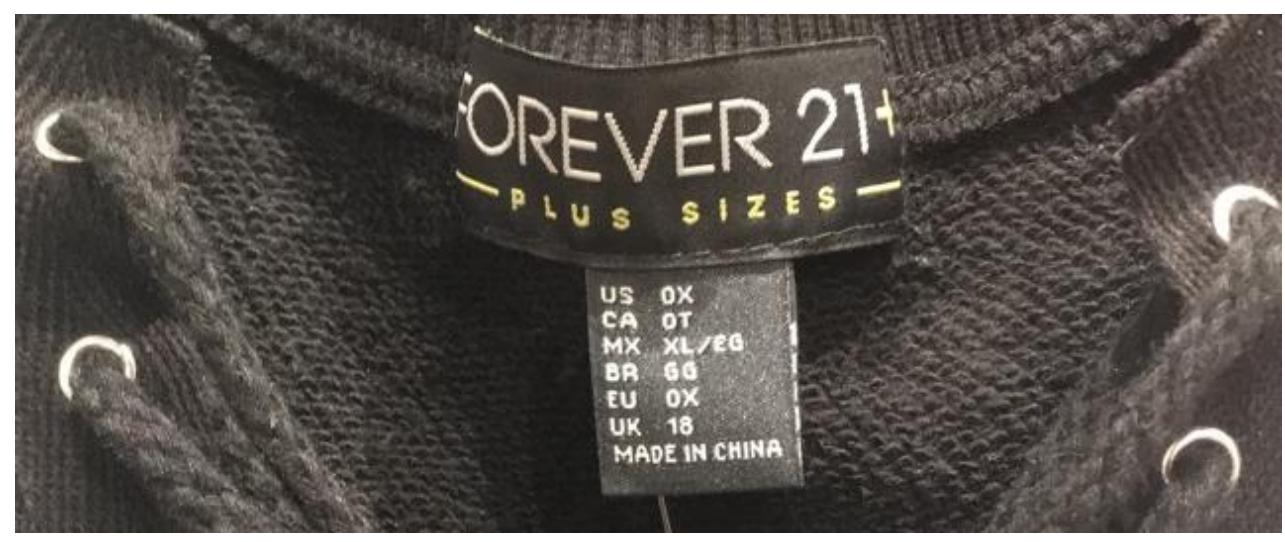

Figure 18. Image of a tag in the Forever 21 plus size section. The Forever 21 tag on this plus size item is labelled as "Forever 21+" and "plus sizes". Both marks indicate this section is for people within a subset group of the whole. 


\section{Discussion}

This research sought an answer to the question of whether or not Athens, Greece, communicated body positivity to women through imagery and clothing, and it was discovered that there was little to no body positive communication present.

Nearly all of the models and mannequins recorded were in the unmarked, ideal size. This is an issue because the only group being represented are women in the unmarked, ideal group. Because informational social influence informs women what is socially acceptable through imagery and other means, imagery of solely unmarked women could communicate to women that the ideal body size is the only size that is socially acceptable.

Combined with social marking, the unmarked size group is seen as natural through the preferred use of ideal-sized women in images, and, merely because other sizes are not seen, the marked size group is considered unnatural.

At the same time, normative social influence causes individuals to conform to what is acceptable and natural. Thus, seeing only ideal sizes could cause individuals to conform to the ideal size to be liked or accepted, and the manner in which they conform could be appearance-focused and unhealthy such as anorexia, bulimia, or restrictive dieting.

Further, most of the clothing sizes recorded were in the small to medium size range. This likely meant that this range had the highest number of items in the store for customers to purchase.

It's worth noting that the higher number of smaller sizes could be because larger sizes are purchased by customers quicker or purchased less by the actual store when it is filling its stock orders. The lack of larger sizes coupled with front-of-store imagery that shows only this size may show women that this size is preferred and socially acceptable. It shows women it is acceptable through informational social influence and, again, could cause them to conform, and conform in unhealthy ways, through normative social influence.

At the same time, marked, plus size groups were not represented in images, and sizes in stores favoured lower sizes. This insinuates a sense of abnormality and a social divide between "normal" people and "plus size" people. 
As a whole, the lack of representation could be communicated to plus size people as size-discrimination, and this is troublesome when considering the ramifications of negative body communication, such as increased eating or poor eating habits.

There was a paradox found in some of the locations where larger sizes were limited through lower size ranges or lower availability for purchase. For example, the H\&M, Nike, and Admiral Sport Short workout pants catered to smaller sizes. This fact is counterintuitive because overweight and obese women also need access to workout clothes if they want to be healthy.

Other times, items seemed to suggest they were unfit for larger sizes through having a lower size range. Certain items, such as short denim shorts for example, had lower size ranges compared to other items. Moreover, the shorts available in the plus size section were vastly different from the other sections in the store. This suggests that this item is only acceptable for smaller sizes, which furthers the social divide between the "normal" and "plus size" group.

The location for the plus size section in Forever 21 also implied size-discrimination as it was on the top floor in a corner next to the athletic clothing section. This placement is problematic because it suggests that plus size clothes need to be out of sight and also insinuates that overweight and obese persons need to work out (even though there are no plus sizes in that section).

In truth, the plus size section as a whole communicates size-discrimination through social marking. The regular section is unmarked, and the plus size section is marked, demarcated from the "normal" clothing. As noted in the literature review, this gives the impression that the plus size section is both "lesser" and "abnormal". Some stores also chose to mark their clothing with literal plus-sized identifiers, indicating to women that they are a part that marked, "subset" group.

Size discrepancies in store and between stores are equally as troublesome as individuals in any size category, not just plus size, may experience the ramifications of sizediscriminatory communication. In other words, people of all sizes could feel shame while shopping if sizes between items, despite the tag being the same, are in-fact different sizes. As an example, a woman who knows she's a size extra-small to small in most stores but can't fit smaller than a size medium or even large in the store she is shopping inside of may leave the store feeling unhappy about herself. 
As labelling theory posits, a person can adopt the persona of a label used to describe them, and any of the situations above, could cause a person to adopt a harmful label onto himself or herself.

The literal separation from "normal" size to plus size can also cause someone to label himself or herself. Moreover, if a person is unable to conform to body standards naturally, normative social influence could presumably cause them to conform unnaturally through eating disorders.

Overall, the ramifications of negative body communication, the social marking divide, and labelling theory can be reduced through body positive communication, and body positive communication could be enhanced by including a variety of body sizes in imagery and in clothing for purchase.

\section{Recommendations}

There are many recommendations that could be made based on this research. First, societies could also look at health education. It is recommended that universal, annual nutritional food education classes in the elementary to high school curriculum are introduced and or developed. Nutritional courses would provide individuals with the skills to make appropriate nutritional choices in the onset.

In a study by Messelink (2016), it was determined that formal education courses on nutrition were lacking; inconsistent labels on food packages were difficult to understand, and many individuals learned about diet at home (pp. 1-15). To improve nutritional communication, Messelink recommended that "formal nutritional guidelines... be reinforced in educational systems... to encourage healthy habits at a young age" (p.13).

Moreover, it is important that body positive communication be introduced early in life because "children as young as three years describe overweight children as 'mean,' 'stupid,' 'lazy,' and 'ugly,' and obese children are 1.6 times” times as likely to be bullied as children who are not overweight" (Lumeng et al., as cited in Tomiyama \& Mann, 2013, p. 4). This fact, when combined with labelling theory, presents that children could develop damaging labels at a young age and could therefore adopt the behaviour for that label early in life.

It is recommended that communication outside and inside clothing stores is improved. Outside store body positive communication could be enhanced by diversifying the sizes of the models and mannequins for customers to see, and in-store body positive communication could be made better by removing all markings denoting abnormality and 


\section{A. Camarneiro}

thus removing the plus size section and tag. Clothing should be displayed by style then by size so that all women go to the same place regardless of size and subset groups are not created because of size.

To that end, universal sizing is clearly required. Sizes between stores and even in stores are vastly different, and women should not feel body shamed simply by trying on a different shirt. This particular aspect may seem inconsequential; however, immense size differences in clothing body shames people of all sizes, meaning more individuals could be impacted by the consequences of negative body size communication.

Stores that cater to subset group sizes also increase the social divide based on body size; therefore, adding larger size ranges would be helpful so that plus size individuals are not divided by store. In this instance, adding these sizes would benefit stores as it was discovered that annual sales for plus size clothing (14 US and up in this study) "is up 17 percent to $\$ 20.4$ billion” (NDP Group, as cited in McGregor, 2016, para. 2).

It is also recommended to maintain a consistent size range for all items, opposed to lowering the size range for certain items and thereby implying that the item is not appropriate for larger persons.

Last, social marking causes a divide between the two groups. To reduce the impression of a social divide and ultimately discrimination, a suggested approach is to modify how the divide—the "us" versus "them" aspect—is perceived by the discriminator, and promote "a common identity... to which both the in-group and outgroup members belong” (Aronson et al., 2013, p. 386). This can be done by introducing more size diversity in imagery and clothing stores.

\section{Conclusion}

Greece's overweight and obesity rate is rising, which implies that Greece's current approach to remedying this issue is not working and a new approach is needed. Societal factors and appearance-focused communication influences consumption and activity, and negative body communication can result in increased or poor consumption and inactivity. Through a review of front-of-store female models and mannequins and the sizes available in clothing stores, it was determined that Greece communicates exclusivity and could improve its body positive communication. Size-diversity in imagery, increasing size ranges, and eliminating plus size sections in stores are a few ways societies can create a more inclusive environment and ultimately work towards creating a healthier population overall. 
30 A. Camarneiro

* Author: Ashley Camarneiro is a recent graduate of MacEwan University's Bachelor of Communication Studies and is now a Marketing Writer. Ashley is a passionate bumanitarian who aims to carry on researching and writing about body positive communication, emotional sustainability, and other similar topics.

\section{References}

Aronson, E., Wilson, T.D., Fehr, B., \& Akert, R.M. (2013). Social Psychology (5th Ed.). New Jersey, USA: Pearson Education, Inc.

American Psychological Association. (2006, February 23). Americans engage in unhealthy behaviors to manage stress [Press release]. Retrieved from http://www.apa.org/news/press/releases/2006/01/stress-management.aspx

Brekhus, W. (1996). Social marking and the mental coloring of identity: Sexual identity construction and maintenance in the United States. Sociological Forum, 11(3), 497522. Retrieved from https://www.jstor.org/journal/sociforu

Cwynar-Horta, J. (2016). The commodification of the body positivity movement on Instagram. Stream: Culture/Politics/Technology, 8(2), 30-56. Retrieved from http://journals.sfu.ca/stream/index.php/stream/article/view/203

Dance On. (2017, August 15). Body-positive dancer Amanda LaCount shows us how she's \#Breakingthestereotype: \#Dancedocs [Video file]. Retrieved August 23, 2017, from https://www.facebook.com/danceonnetwork/videos/1391467604241203/

Dubecky, M. (n.d.). These 6 brands are embracing what real bodies look like-and we're loving every second. Bust. Retrieved from http://bust.com/style/13791-these-6brands-are-embracing-what-real-bodies-look-like-and-we-re-loving-everysecond.html

Engeln, R. (2017). Enough talk of bikini bodies: If you care about fitness and women, never use the term "bikini body" again. Psychology Today. Retrieved from https://www.psychologytoday.com/blog/beauty-sick/201707/enough-talk$\underline{\text { bikini-bodies }}$ 
31 A. Camarneiro

Forever 21. (2017) [Web site]. Retrieved from

http://www.forever21.com/CA/Product/Category.aspx?br=f21\&category=app -main\&lang=en-US

Fraser, S. (2017, April 17). Can body positivity be too positive? A P.E.I. woman asks 'I'm all over loving my body and saying it's okay to be big when I want to eat that bacon cheeseburger'. CBC News. Retrieved from http://www.cbc.ca/news/canada/prince-edward-island/pei-body-positivitytammy-mackinnon-christine-mcnearny-1.4068138

Gordon, E. (2017, August 17). Re: Body-positive dancer Amanda LaCount shows us how she's \#Breakingthestereotype: \#Dancedocs [Facebook comment]. Retrieved from https://www.facebook.com/danceonnetwork/videos/1391467604241203/

H\&M. (n.d.). [Web site]. Retrieved from http://www2.hm.com/en_ca/women.html

Kościński, K. (2013). Attractiveness of women’s body: Body mass index, waist-hip ratio, and their relative importance. Behavioral Ecology, 24(4), 914-925. DOI:

10.1093/beheco/art016

Love, J. (2017, August 16). Re: Body-positive dancer Amanda LaCount shows us how she's \#Breakingthestereotype: \#Dancedocs [Facebook comment]. Retrieved from

https://www.facebook.com/danceonnetwork/videos/1391467604241203/

Close, H. M. (2017, June 9). Re: This girl started a fashion line after being bullied [Facebook comment]. Retrieved from https://www.facebook.com/NowThisHer/videos/1058491354281739/

Mann, T., Tomiyama, A.J., \& Ward., A. (2015). Promoting public health in the context of the "obesity epidemic": False starts and promising new directions. Perspectives on Psychological Science, 10(6), 706-710. Retrieved from http://journals.sagepub.com/doi/pdf/10.1177/1745691615586401

McGregor, L. (2016, September 9). Most of the apparel industry is missing out on a $\$ 20$ billion opportunity. Sourcing Journal. Retrieved from https://sourcingjournalonline.com/plus-size-20-billion-opportunity/ 
Messelink, M. (2016). Nutritional communications across climates: A comparative research study between Ecuador and the Netherlands. Earth Common Journal, 6(1), 1-15. Retrieved from https://journals.macewan.ca/earthcommon/article/view/883

Movement. (2017). In Oxford Learner's Dictionaries. Retrieved from http://www.oxfordlearnersdictionaries.com/definition/english/movement?q=m ovement

Murray, J.L., Linden, R., \& Kendall, D. (2011). Sociology in our times (5th ed.). Toronto, ON: Nelson Education.

NowThis Her. (2017, June 9). This girl started a fashion line after being bullied. In Facebook [Video file]. Retrieved August 23, 2017, from https://www.facebook.com/Now'ThisHer/videos/1058491354281739/

Plus-size. (2017) In English Oxford Living Dictionaries. Retrieved from https://en.oxforddictionaries.com/definition/us/plus-size

Puhl, R. \& Brownell, K.D. (2001). Bias, discrimination, and obesity. Obesity, 9(12), 788805. DOI: $10.1038 /$ oby.2001.108

Relationship Rules. (2017, August 22) Couple loses weight together [Video file]. Retrieved August 23, 2017, from https://www.facebook.com/RelationshipRules/videos/1720562674683258/

Reber, A. S., Allen, R., \& Reber, E. S. (2009). Marked-unmarked adjectives. The Penguin dictionary of psychology (4th ed.). Retrieved from http://ezproxy.macewan.ca/login?url=http://search.credoreference.com/conte nt/entry/penguinpsyc/marked_unmarked_adjectives/0?institutionId=2632

Schreiber, K. \& Hausenblas, H. (2016, August 11). What does body positivity actually mean: How (and why) the concept continues to be misunderstood. Psychology Today. Retrieved from https://www.psychologytoday.com/blog/the-truth-aboutexercise-addiction/201608/what-does-body-positivity-actually-mean

Tali, D. (2016, September 30) “The 'average' woman is now size 16 or 18 . Why do retailers keep failing her?" Forbes. Retrieved from https://www.forbes.com/sites/didemtali/2016/09/30/the-average-womansize/\#2af891e22791 
33 A. Camarneiro

Tomiyama, A.J. \& Mann, T. (2013). If shaming reduced obesity, there would be no fat people. The Hastings Center Report, 43(3), 4-5. DOI: http://dx.doi.org/10.1002/hast.166

Wimmer, R.D., \& Dominick, J.R. (2014). Mass media research: An introduction (10th ed.). Belmont, CA: Thomson Wadsworth.

World Health Organization. (2013). Nutrition, physical activity, and obesity: Greece [PDF]. Retrieved from http://www.euro.who.int/_data/assets/pdf_file/0013/243301/Greece-WHOCountry-Profile.pdf 